\title{
The Feasibility of Using Probiotics to Treat Depression
}

\author{
YU XIHE \\ ${ }^{1}$ University of Warwick, Coventry, United Kingdom, CV4 7AL
}

\begin{abstract}
Depression is one of the most common mental disorders. It refers to a kind of mood disorder with significant and lasting depression as the main clinical symptoms caused by various reasons. Its core symptoms are depression, loss of interest, lack of energy, often accompanied by physical symptoms, cognitive symptoms and so on. Serious patients have the risk of self injury and suicide. Most drug treatments focus on changing neurotransmitter activity in the brain, and Probiotics are a kind of active microorganisms which are beneficial to the host. They are colonized in the human intestinal tract and reproductive system and can produce exact health effects, thus improving the balance of the host's microecology and playing a beneficial role. Ingestion of certain probiotics seems to be a potential treatment for depression.
\end{abstract}

\section{INTRODUCTION}

Depression is a type of mood disorder whose main clinical features are significant and lasting depression, delayed thinking, decreased willpower, cognitive impairment, and physical symptoms. It has a high incidence, high recurrence, high suicide rate and high disability rate and other characteristics[2]. According to reports, the global prevalence of depression has reached $4.4 \%$, and the number of depression patients has reached 322 million [3]. According to statistics from the World Health Organization (WHO) in 2017, depression affects more than 300 million people worldwide and about 800,000 people with depression commit suicide every year. Although a series of effective antidepressants have been developed, for major depression, more than $50 \%$ of patients do not affect the first antidepressant prescribed by a doctor [4], even if they receive multiple treatments. But there are still $30 \%$ of patients ineffective; In addition, even if the traditional treatment can relieve the condition of some patients, these patients will also be accompanied by some dysfunction. Commonly used treatments for depression not only have a high rate of ineffectiveness and slow onset, but also cause serious discomfort and side effects. These treatments not only make depression patients unable to work normally, but also bring serious economic burdens to patients and reduce the quality of life[6]. This article starts with the influence of intestinal flora on depression and possible mechanisms and aims to explore the preventive and therapeutic effects of changes in intestinal flora on depression, providing more effective and less costly treatment with less side effects for patients with depression in the future.

\section{ANALYSIS}

\subsection{Overview of Gut Flora}

The human intestine is a nutrient-rich environment with as many as 100 trillion microorganisms. Normal human intestinal microbes are mainly composed of two categories, namely the Bacteroides and Firmicutes, which account for more than $3 / 4$ of the microbial community[7,9]. There are also relatively small numbers of Proteobacteria, Actinomycetes, Defective Microbacteria and Fusobacteria. Although the mechanism of the relationship between the intestinal flora and human health is mostly unknown, healthy individuals tend to have richer microbial diversity [10].

\subsection{The influence of intestinal flora on depression}

Studies have shown that the abundance and diversity of the intestinal flora of patients with depression are quite different from that of healthy people, and it is specifically higher in the proportion of Actinomycete, Bacteroides, and Proteobacteria in depression patients than that in healthy people. Using 16S rRNA gene sequence analysis technology to compare the fecal microbial composition of 46 depression patients and 30 healthy controls, it was found that the fecal flora of depression patients' diversity (Shannon index, Simpson index and Evenness index) was significantly increased, the expression abundance of Enterobacteriaceae increased, and the abundance of Faeculus genus decreased [11]. Another study found that the daily diversity of intestinal flora (the degree of similarity in the

* Corresponding Author's Email: xihe.yu@outlook.com 
development of microbial systems) is also significantly different between patients with depression and healthy people[12].

Kelly et al. [13] analyzed the microbial community, kynurenine, tryptophan, and cytokines in 34 depression patients and 33 healthy controls. The study found that compared with healthy controls, the diversity, abundance and composition of the gut bacteria in the depression group were all changed. The ratio of kynurenine to tryptophan was significantly higher than that of the control group, and the level of interleukin stone was also significantly increased.
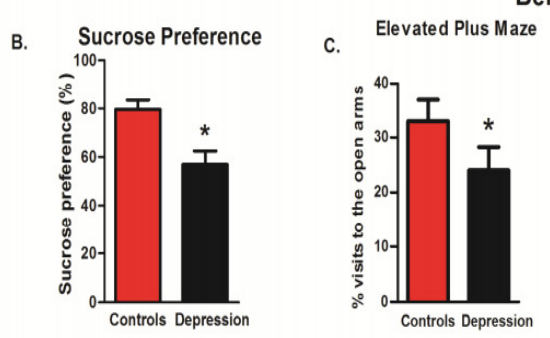

Behavioural Profile
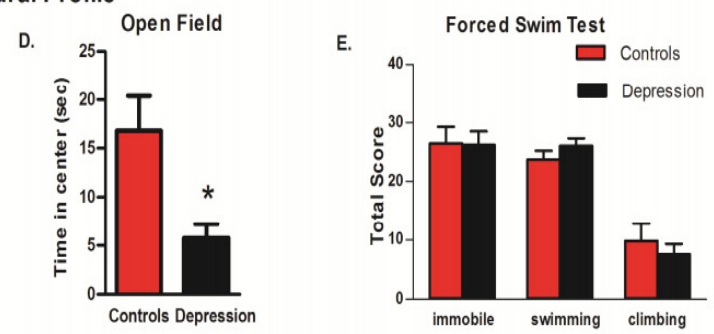

Physiological Profile
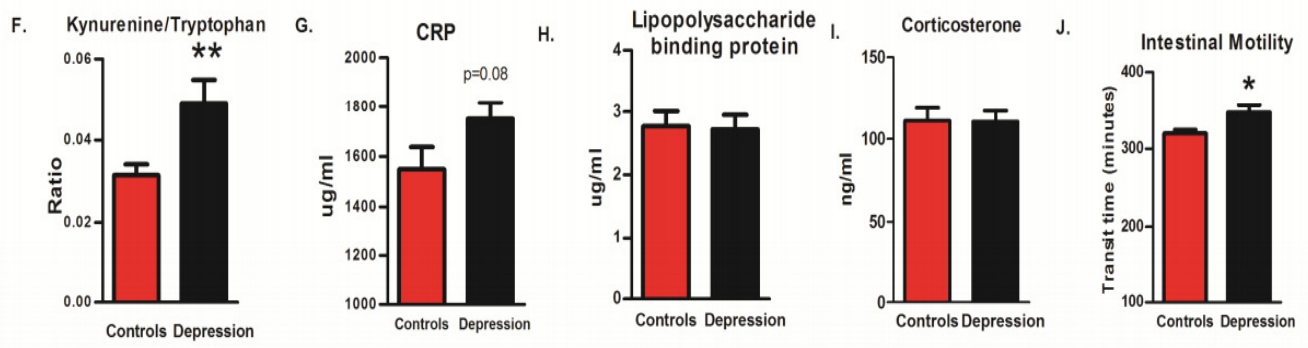

Figure 1. Behavioural and Physiological Profile

The fecal flora of depression patients and healthy controls were transplanted into the deficient intestine in rats with tract microflora. It was found that rats receiving fecal microbiota from depression patients showed anhedonia-like behaviors (depression-like symptoms), as well as obviously elevated kynurenine levels, tryptophan metabolism disorders, and intestinal. The decrease in the abundance of tract flora indicates that changes in the intestinal flora can indeed induce depression symptoms (Figure 2).

Altered Gut Microbiota Richness \& Diversity following fecal transplantation

A.

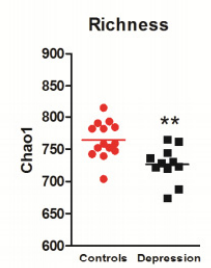

B.

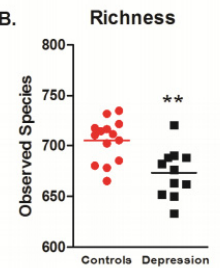

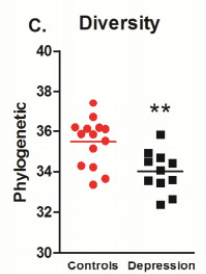

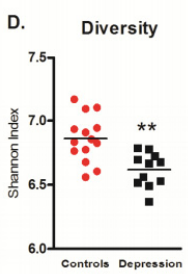

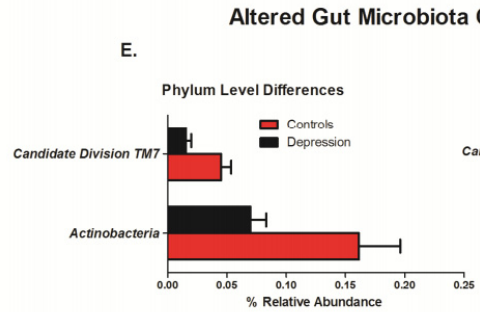

G.

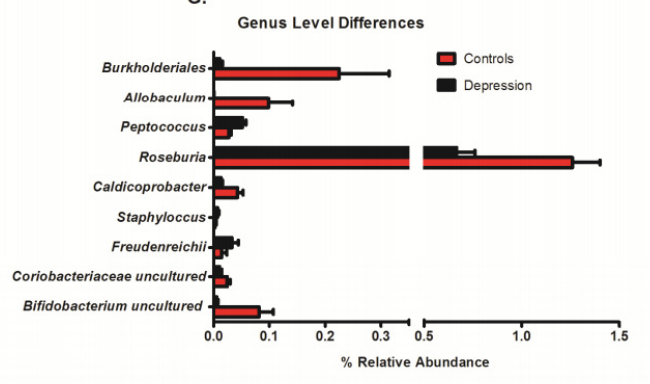

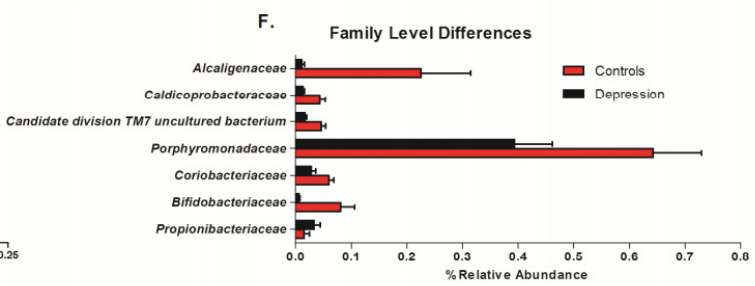

H.

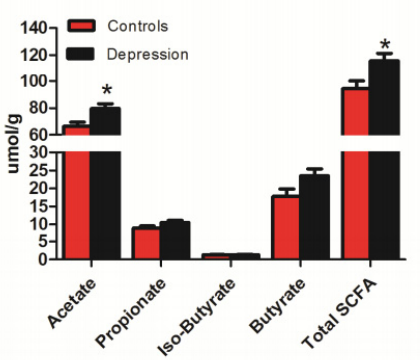

Figure 2. Changes in the intestinal flora 
Zheng et al. [14] also obtained similar results and found that the behavior and flora of mice receiving fecal flora transplantation from depression patients are consistent with depression patients who provide fecal microbes, indicating that the intestinal flora can cause depression symptoms by affecting the body's metabolism.

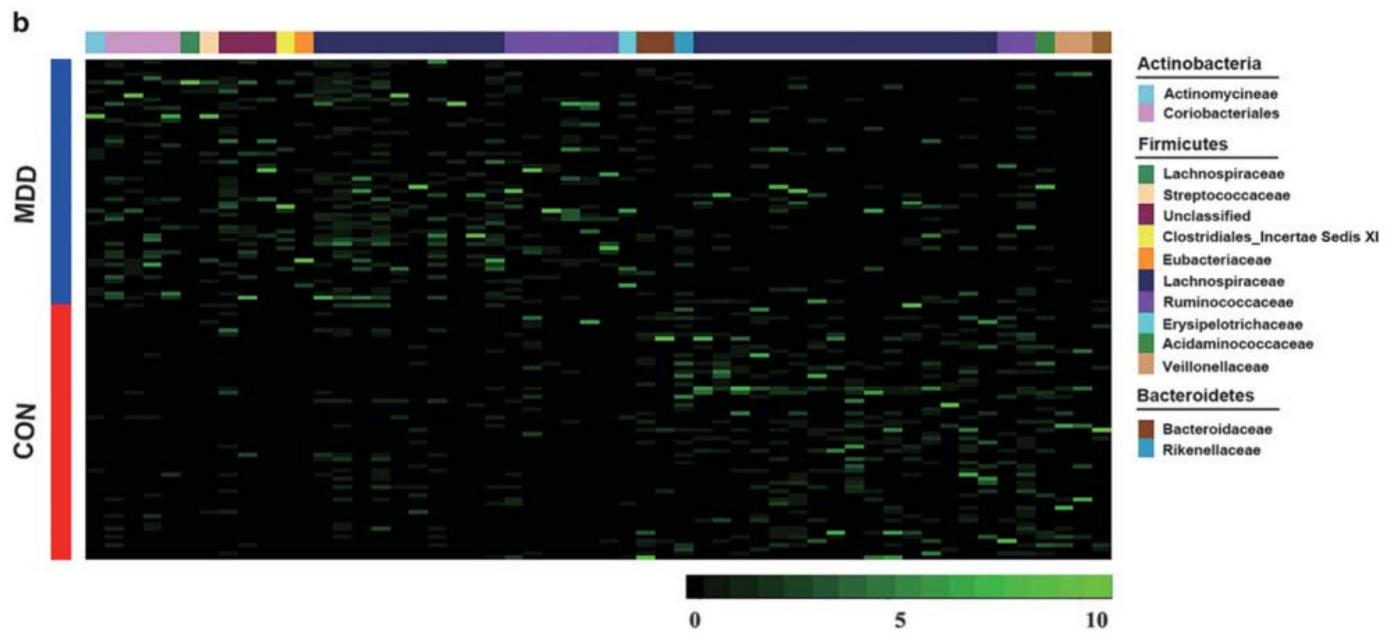

Figure 3. (b) Heatmap of the 54 discriminative operational taxonomic units (OTUs) abundances between depressed subjects and healthy controls.

The taxonomic assignment of each OTU is provided on the right. The increased OTUs in MDD subjects are arranged on the left, and the decreased OTUs are arranged on the right. As the changed directions of some discriminative OTUs sharing the same taxonomic assignment at the class level were not identical, there are some colors that are repeated across the same axis.

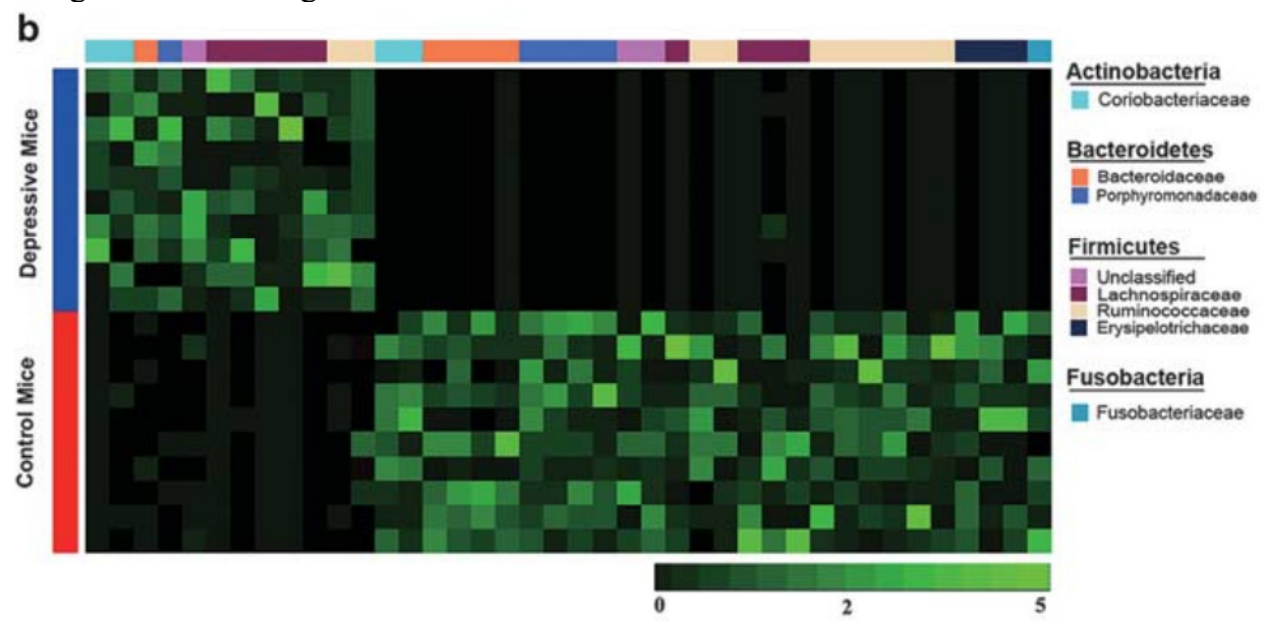

Figure 4. (b) Relative abundance of operational taxonomic units (OTUs) responsible for discriminating depressed mice from control mice.

The taxonomic assignment of each OTU is provided on the right. The increased OTUs in depressed mice are arranged on the right, and the decreased OTUs are arranged on the left.

Nishino et al. [15] used germ-free mouse offspring and germ-free mice that were inoculated with Bifidobacterium infants $(\mathrm{Bi})$ and Blautia coccoides $(\mathrm{Bc})$ as the research objects. Conducted behavioral tests in China and found that at all time points, there was no significant difference in DT30 (a parameter of anxiety and depression-like behavior) between $\mathrm{Bc}$ mice and sterile mice, and the DT30 level of Bi mice decreased significantly. This result indicates that different strains have different behavioral phenotypes on the host. 


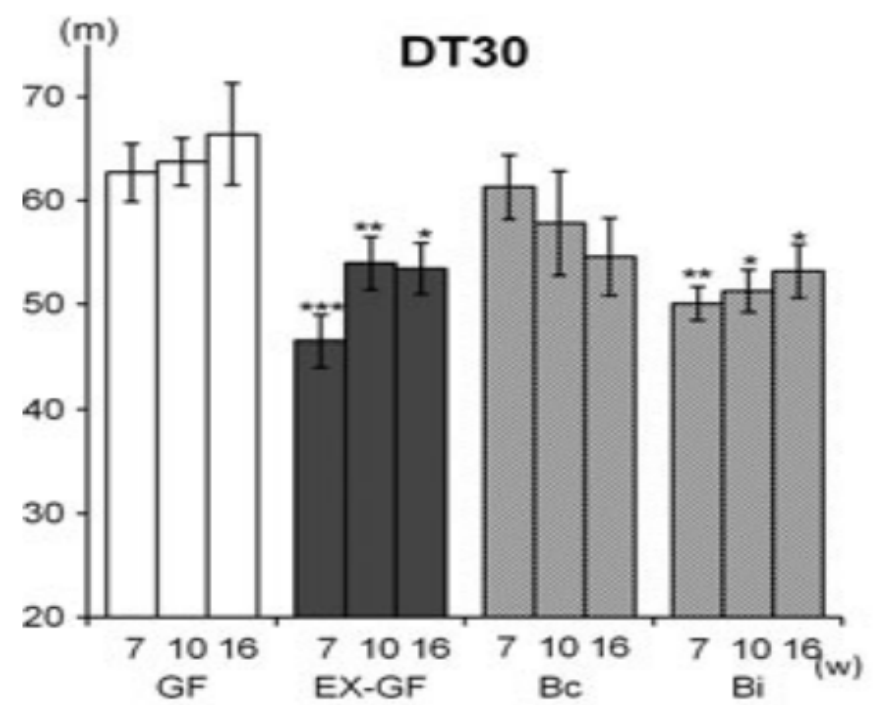

Figure 5. Effects of commensal microbiota on behaviors of the host.

In figure 5, GF, EX-GF, Bc, and Bi mice at the age of 7, 10, and 16 weeks were subjected to OP and MB tests, and DT30 was evaluated as described in the methods. All data are expressed as the mean SE ( $\mathrm{n}=14-19$ per group). $* \mathrm{P}<0.05, * * \mathrm{P}<0.01$ and $* * * \mathrm{P}<0.001$ were significantly different from the corresponding GF values.

\subsection{Microbes in Brain-gut-axis mechanism}

By far, studies have shown that symbiotic microorganisms do affect the nervous function of the host, and affect mood and behavior (depression, anxiety, social behavior, partner choice). The specific mechanism is still unclear. There are three situations (Figure 6):

\section{Pathways linking the microbiome and central nervous system}

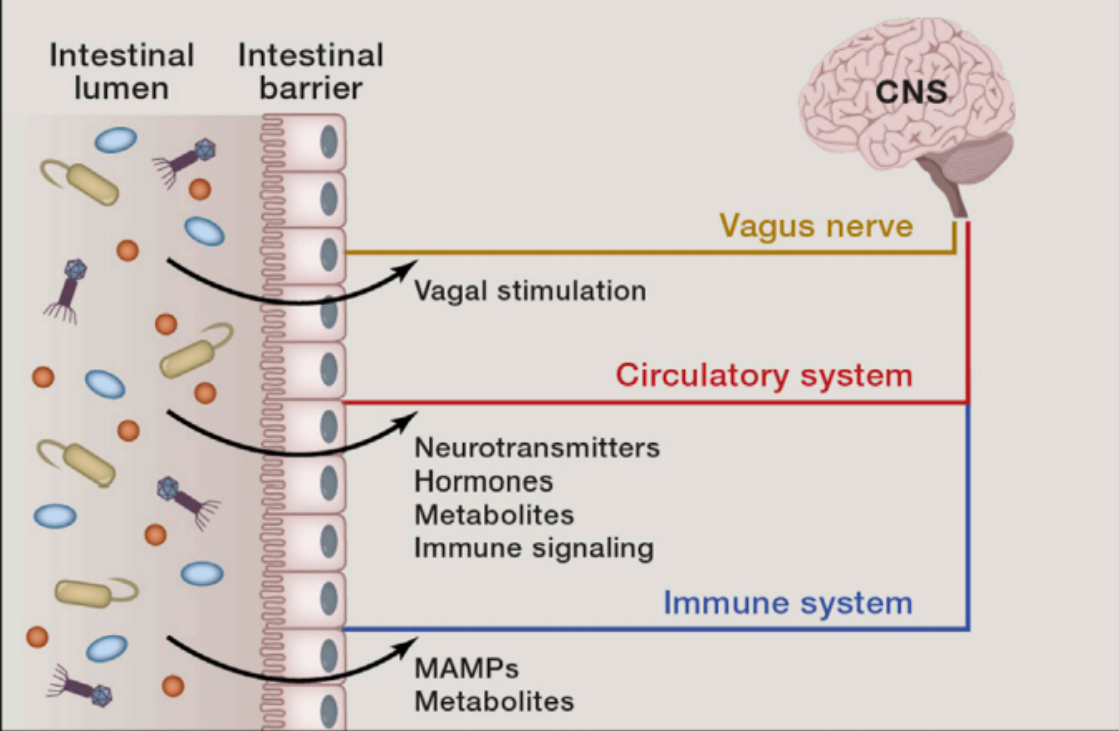

Signals from the intestinal microbiome may potentially traffic to the CNS via several mechanisms: (1) direct activation of the vagus nerve from the ENS to the GNS; (2) production of, or induction of, various metabolites that pass through the intestinal barrier and into the circulatory system, where they may cross the BBB to regulate neurological function; (3) and MAMPs (such as LPS, BLP, and PSA) and metabolites produced by the microbiota can signal to the immune system. Immune cells (and particularly their cytokines) can influence neurophysiology.

Figure 6. Pathways Linking the Microbiome and CNS 
- Producing or inducing the production of various metabolites (metabolic precursors of hormones, neurotransmitters, or active metabolites themselves) pass through the intestine. The tract barrier enters the circulatory system and then may pass through the blood-brain barrier to regulate nerve function.

- Symbiotic microorganisms affect the systemic immune system by producing MAMPs (microbial related molecular patterns, such as lipopolysaccharide LPS, bacterial lipoprotein BLP, PSA) and immune cells (especially their cytokines) can affect the nervous system.

- The enteric nervous system ENS can be directly connected to the central nervous system through the vagus nerve, providing a direct neural pathway for intestinal microbial signals to be transmitted to the brain.

The interaction between the host and its microbiota is very complex. Intestinal microbes affect many aspects of metabolism, producing hormones and their metabolic precursors. As shown in Figure 6, neurotransmitters may directly produce active metabolites themselves [16]. Symbiotic bacteria also have the ability to affect the state of the systemic immune system, which may change the way the immune system subsequently interacts with the nervous system [17]. In addition, the enteric nervous system (ENS) is directly connected to the central nervous system (CNS) through the vagus nerve, providing a direct neurochemical pathway for the transmission of signals promoted by microorganisms in the gastrointestinal tract to the brain [18].

According to Figure 7, Microbes may communicate with the nervous system in indirect ways, such as the immune system[19]. Immune signals can cause certain neurological diseases, including neurodegenerative diseases and psychological diseases, such as anxiety and depression. Microorganism-related molecular patterns (MAMPs) activate various cells of the immune system, and especially innate immune cells such as macrophages, neutrophils, and dendritic cells. Once activated, these cells produce various pro-inflammatory cytokines, such as IL- $1 \alpha$, IL- $1 \beta$, TNF- $\alpha$, and IL- 6 , which pass through the $\mathrm{BBB}$ through diffusion and cellular transport. After reaching the brain, these cytokines will act on the receptors expressed by neurons and glial cells, and change the active and physiological states of the receptors.

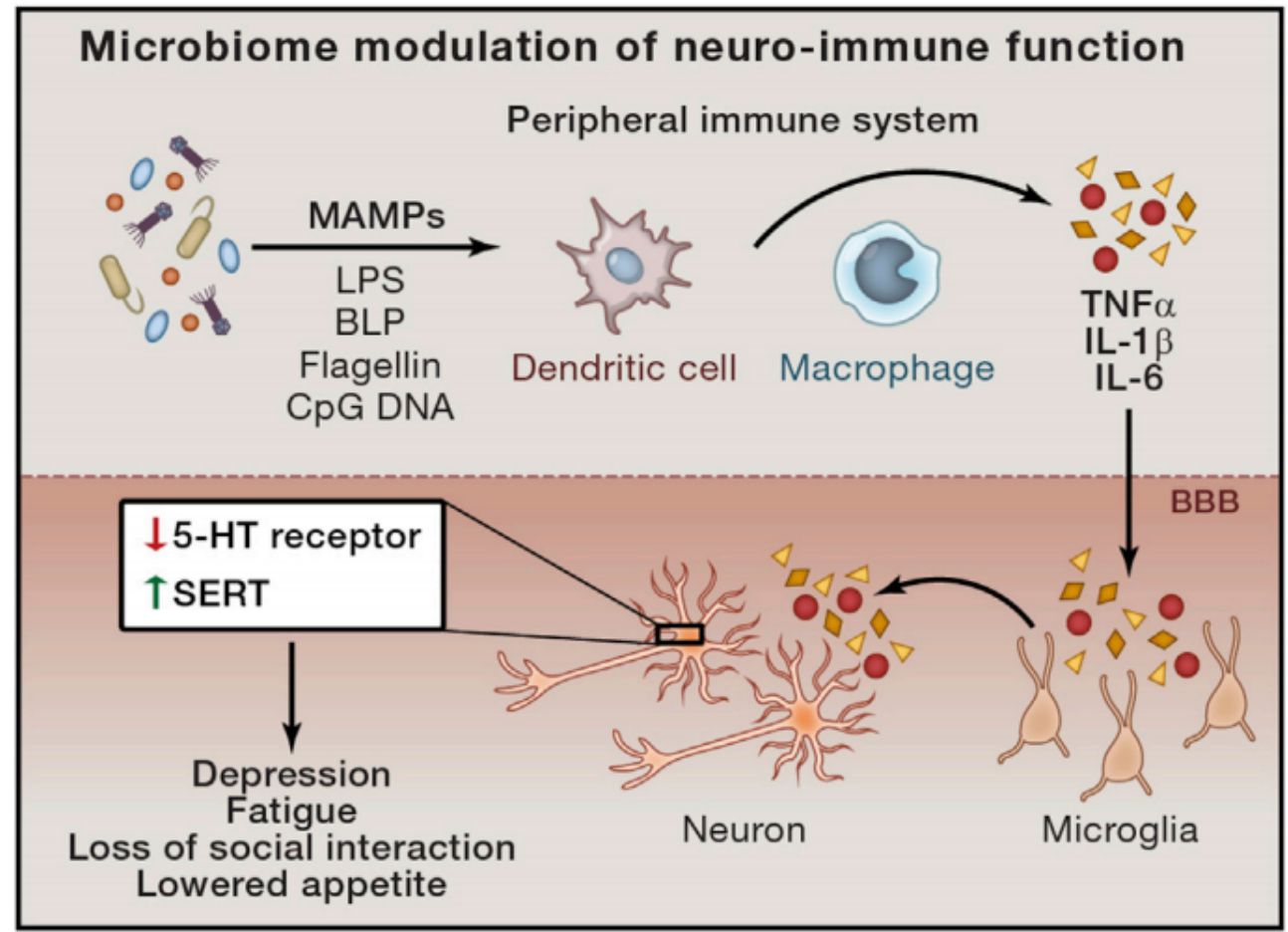

Figure 7. Microbiome Modulation of Neuro-Immune Function

Contrary to conventional wisdom, the content of most neurotransmitters in the intestine is equal to or exceeds the level in the brain. In addition, the total content of various neurotransmitters in the intestine is higher in the human body than in the brain. Although most of the current data on microbial regulation of neurotransmitter production and/or levels can be used in the intestine and the surrounding environment, the regulation of local neurotransmitters by intestinal bacteria may have long-distance effects on the brain. Neurotransmitters or other molecules derived from gut microbes may regulate the activity of the vagus nerve, which is the main nerve that connects the ENS to the CNS and subsequently affects brain function [20]. Microbial-derived metabolites which may act as precursors for neurotransmitter production such as tryptophan and cross the intestinal barrier and BBB may also subsequently affect the concentration of neurotransmitters throughout the body and central nervous system. 


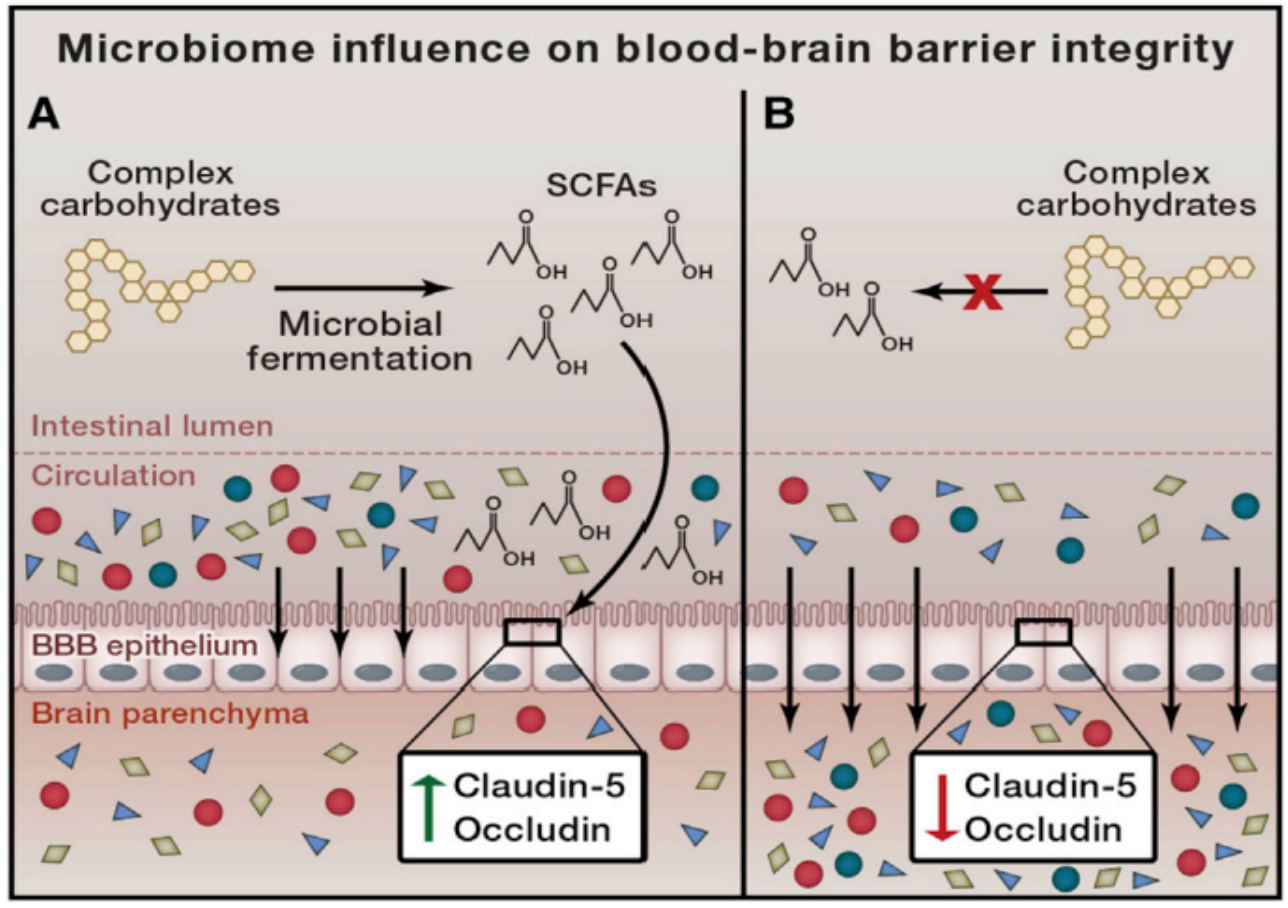

Intestinal microbes are capable of fermenting complex carbohydrates into SCFAs.

(A) Microbially produced SCFAs signal to endothelial cells that create the BBB and increase production of the tight junction proteins claudin-5 and occludin. This leads to a tight and selective barrier, preventing undesired metabolites from entering the brain parenchyma.

(B) In the absence of microbial fermentation, no SCFA signaling occurs, and tight junction proteins are repressed. This leads to increased permeability of the BBB and a loss of the selective barrier to serum metabolites.

Figure 8. Microbiome Influence on BBB Integrity

\section{The direction of future research}

Although the influence of microorganisms on metabolism and immunity in many aspects has been confirmed, the research on gut microorganisms and the brain needs to continue.

The article emphasizes the application of GF mice in the field of mental illness research. Mice can discover microorganisms that regulate nerves. Research related microorganisms through genetics methods, and study their metabolic pathways, and may find metabolites that regulate the host's neurophysiology, using microbial metabolites as an opportunity to study unknown metabolic pathways of the host. Or new functions of metabolic pathways are known, and these metabolisms may be related to neurological function and behavior.

It is necessary to understand whether the influence of microorganisms on the host occurs during a specific developmental period and then has an irreversible neurological influence (developmental) downstream. or whether it can actively and continuously regulate neurological function (active). The physiological consequences of disease-related microbial changes have to be taken into consideration, and whether the result of microbial colony structure changes is unique. With the continuous deepening of research, we can begin to design a reasonable treatment plan based on microorganisms.

\section{Conclusion}

At present, a large number of studies have confirmed that the intestinal flora and depression are closely related, but the cause and effect of the relationship are still inconclusive. It may be that certain events or stress trigger emotional changes, and these emotional changes subsequently change the gut microbiota. It may also be changed in the gut microbiota caused by digestion. Infection can lead to depression-like behaviors. Therefore, the causal relationship between the two needs to be further explored. In addition to traditional medication and psychotherapy for depression, and intestinal flora therapy is also on the agenda. At present, fecal flora transplantation and probiotic therapy are used to change the intestinal flora, both of which have been proven to be effective in improving individual depression symptoms. However, most of these studies are based on animal experiments such as antibiotic treatment of animals or sterile animals. In view of the genetic differences between rodents and humans, further breakthroughs are needed from animal experiments to popularization and application in the population, and more relevant clinical trials are needed. Research confirms the feasibility of intestinal flora therapy in the population. 


\section{Acknowledgement}

I would like to give my sincere gratitude to Prof. Li, my tutor who, with extraordinary patience and consistent encouragement, gave me great help by providing me with the advice of great value and inspiration of new ideas. It is his suggestions that draw my attention to deficiencies and make my thoughts clearer.

\section{References}

1. Jiang Kaida. Psychiatry[M]. Beijing: People's Medical Publishing House, 2005: 123-124.

2. Liang Shan, $\mathrm{Wu}$ Xiaoli, $\mathrm{Hu} \mathrm{Xu}$, et al. The development and trend of depression research-looking at depression from the bacteria-gut-brain axis[J]. Science Bulletin, 2018, 63 (20): 2010-2025.

3. Su Wenjun, Cao Zhiyong, Jiang Chunlei. Inflammatory mechanism and new strategies for diagnosis and treatment of depression[J]. Acta Physiology, 2017, 69 (5): 715-722.

4. Ruhe HG, Huyser J, Swinkels JA, et al. Switching antidepressants after a first selective serotonin reuptake inhibitor in major depressive disorder:a systematic review[J]. J Clin Psychiatry, 2006, 67(12):1836-855.

5. Ormel J, Oldehinkel AJ, Nolen WA, et al. Psychosocial disability before, during, and after a major depressive episode:a 3-wave Population-based study of state, scar, and trait effects $[\mathrm{J}]$. Arch Gen Psychiatry, 2004, 61(4):387-392.

6. Sivertsen $\mathrm{H}, \mathrm{Bj}\} \mathrm{rkl}\} \mathrm{f} \mathrm{GH}$, Engedal $\mathrm{K}$, et al. Depression and quality of life in older persons:A review [J].Dement Geriatr Cogn Disord, 2015, 40(-6):311-339.

7. Zhang Yi, Zhao Yingying, Zhao Jingjie, et al. Research progress on the influence of intestinal flora on anxiety and depression[J]. Journal of Clinical and Experimental Medicine, 2018, 17 (18 ): 2012-2014.

8. Planchez B, Surget A, Belzung C .Animal models of major depression:drawbacks and challenges[J]. Neural Transm (Vienna), 2019, 126(11):1383-408.

9. Eckburg PB, Bik EM, Bernstein CN, et al. Diversity of the human intestinal microbial flora $[\mathrm{J}]$. Science, 2005, 308(5728):1635-1638.

10. Hollister EB, Gao C, Versalovic J. Compositional and functional features of the gastrointestinal microbiome and their effects on human health [J].Gastroenterology, 2014, 146(6):1449-458.

11. Jiang $H$, Ling $Z$, Zhang $Y$, et al.Altered fecal microbiota composition in patients with major depressive disorder [J].Brain Behav Immun, 2015, 48:186-94.

12. Fang Zheng. Exploring the relationship between depression and intestinal flora through 16S rRNA sequence analysis[D]. Chongqing Medical University, 2016.

13. Kelly, J. R. , Borre, Y., Brien, C. O., Patterson, E., \& Dinan, T. G. . (2016). Transferring the blues: depression-associated gut microbiota induces neurobehavioural changes in the rat. Journal of Psychiatric Research, 82, 109-118.

14. Zheng, P. , Zeng, B. , Zhou, C. , Liu, M. , Fang, Z., $\& \mathrm{Xu}, \mathrm{X}$. , et al. (2016). Gut microbiome remodeling induces depressive-like behaviors through a pathway mediated by the host's metabolism. Mol Psychiatry.

15. Nishino, R. , Mikami, K. , Takahashi, H. , Tomonaga, S. , Furuse, M. , \& Hiramoto, T. , et al. (2013). Commensal microbiota modulate murine behaviors in a strictly contamination-free environment confirmed by culture-based methods. Neurogastroenterology \& Motility, 25(6), 521-e371.

16. Galley, J.D., Nelson, M.C., Yu, Z. et al. Exposure to a social stressor disrupts the community structure of the colonic mucosa-associated microbiota. BMC Microbiol 14, 189

(2014). https://doi.org/10.1186/1471-2180-14-189

17. Belkaid, Y. , \& Hand, T. . (2014). Role of the microbiota in immunity and inflammation. Cell.

18. Johnston, J. , Griffin, S. J. , Baker, C. , Skrzypiec, A. , \& Forsythe, I. D. . (2014). Initial segment kv2.2 channels mediate a slow delayed rectifier and maintain high frequency action potential firing in mntb neurons. The Journal of Physiology, 586, 3493-3509.

19. Dantzer, R. , Konsman, J. P. , Rose-Marie Bluthé, \& Kelley, K. W. . (2000). Immune system. , 85(1-3), 0-65.

20. Bravo, J. A. , Forsythe, P. , Chew, M. V. , Escaravage, E. , \& Cryan, J. F. . (2011). From the cover: ingestion of lactobacillus strain regulates emotional behavior and central gaba receptor expression in a mouse via the vagus nerve. Proceedings of the National Academy of Sciences, 108(38), 16050-16055 